\title{
The effect of ingested alcohol on fusion latency at various viewing distances
}

\author{
R. J. MILLER \\ Washington State University, Pullman, Washington
}

\begin{abstract}
Although diplopia is a frequently reported symptom of severe alcohol intoxication, there have been few past attempts to describe in any systematic fashion the specific impact of alcohol on fusion. In the present experiment, fusion latency was assessed for 8 male emmetropes. At the beginning of the alcohol condition, they consumed a drink containing $1.4 \mathrm{ml} / \mathrm{kg}$ of $95 \%$ ethanol, plus diluent. For the placebo condition, the drink consisted only of diluent. In each condition, fusion latency (the time required to fuse a binocularly visible target) was recorded for targets requiring a magnitude of vergence that varied from +10 through -2 meter angles, in 1-meterangle steps. It was found that the far point of fusion approached the observer as a direct function of intoxication level. Furthermore, fusion latency increased for optically far and near targets as a systematic function of intoxication, with no effect of alcohol on intermediate targets. The results were consistent with previous findings of alcohol-induced narrowing of vergence range, suggesting that alcohol causes the farthest and nearest distances at which fusion latency is affected to approach an intermediate value. Measurable effects on fusion latency were observed with blood alcohol levels as low as $0.05 \%-0.06 \%$, considerably below the legal limit for operating a motor vehicle in most states.
\end{abstract}

One of the symptoms of severe ethanol intoxication is diplopia. Diplopia occurs when retinal disparity exceeds the capacity of sensory fusion. Under normal circumstances, motor fusion produces sufficient vergence to reduce disparity until it lies within Panum's area. The sensory fusion mechanism then fuses the retinal images and diplopia is eliminated. Alcohol-induced inability to produce fusion could, in theory, reflect alcohol effects on motor fusion, sensory fusion, or both.

Most of the relevant available literature emphasizes the effect of alcohol on vergence and related processes (e.g., Adams, 1978; Brecher, Hartman, \& Leonard, 1955; Cohen \& Alpern, 1969; Colson, 1940; Hogan \& Linfield, 1983; McNamee, Piggins, \& Tong, 1981; Miller, Pigion, \& Martin, 1985; Miller, Pigion, \& Takahama, 1986; Moskowitz, Sharma, \& Shapero, 1972; Powell, 1938; Wilson \& Mitchell, 1983; Zuschlag, 1965). A consistent general finding is that alcohol reduces the available range of vergence. That is, alcohol induces decreased divergence for far viewing and decreased convergence for near viewing; the near and far limits are reduced.

This investigation was supported in part by funds provided for medical and biological research by the State of Washington Initiative Measure No. 171. Selected portions of this experiment were presented at the 31st annual meeting of the Psychonomic Society, New Orleans, November 16, 1990. I thank Steven Meier, R. J. Densley, Jenny Fitzmorris, Debbie Metzler, Steven Raab, Annette Taylor, and Michael Donnelly for technical assistance rendered during various phases of this investigation. Correspondence should be addressed to R. J. Miller, 209 Johnson Tower, Department of Psychology, Washington State University, Pullman, WA 99164-4820.
Although the studies above have been useful in describing the effect of alcohol on vergence, they have not for the most part been direct tests of its effect on diplopia. Fusional ability can be represented in a number of ways. Three that are of practical interest are the near and far points of fusion and fusion latency.

The near point of fusion is the minimum viewing distance (or maximum vergence angle) at which fusion can be attained. In view of alcohol's tendency to narrow vergence range, one might expect near point distance to increase as a function of intoxication level. A few studies have provided some description of the effect of alcohol on the near point of fusion (e.g., Brecher et al., 1955; Hogan \& Linfield, 1983; Powell, 1938; Wilson \& Mitchell, 1983). These have mostly involved fairly crude measures in which a vertical line or rod is moved toward the observer until the observer can no longer fuse it. Although it is difficult to determine precise details from most of these reports, their results all seem to indicate an outward shift in near point with alcohol intoxication.

The far point of fusion is the maximum viewing distances (or minimum vergence angle) at which fusion can be attained. In view of alcohol's effect on vergence range, one might expect that the distance of the far point of fusion would decrease as a function of intoxication. The far point of fusion has not attracted much interest in alcohol research, but some measures of fusional reserve have been reported. Fusional reserve is assessed by providing a binocularly viewed (usually far) target. Base-out or base-in prisms are inserted, and their power is increased until fusion is no longer attainable. The maximum divergence (abduction) or convergence (adduction) that can be pro- 
duced without loss of fusion is taken as a measure of the far or near fusional reserve. With a far target, the maximum abduction is essentially equivalent to the far point of fusion.

Moskowitz et al. (1972) found that alcohol produced a reduction in maximum abduction of 2.5-2.7 prism diopters (PD). Seedorff (1956) reported alcohol-induced reductions in abduction of $50 \%$ or more that were highly correlated with blood alcohol level (BAL). ${ }^{1}$ In contrast, Colson (1940) reported that alcohol produced a decrease in adduction, with no change in abduction. However, his conclusion was based on only 3 subjects and an unconventional representation of duction. In terms of more conventional duction measures, his results would appear to be very similar to those of Moskowitz et al. (1972) and Seedorff (1956), with alcohol-induced decreases in abduction of about 6 PD.

Fusion latency (also sometimes called fusion speed, fusion time, or fusion power) is the amount of time required to attain fusion. Reduced fusional ability can be reflected in an increase in fusion latency even when there is sufficient fusional ability to overcome diplopia. One might expect that as alcohol intoxication reduces the vergence range, fusion latency would increase for far and near targets. Furthermore, if there is no sensory fusion involvement, there should be less or no change in fusion latency at intermediate viewing distances. On the other hand, if alcohol-induced diplopia affects sensory fusion, one might expect fusion latency to be affected even at intermediate viewing distances.

There is little direct evidence regarding the effect of alcohol on fusion latency. Brecher et al. (1955) reported that fusion latency for targets at three distances $(600,82$, and $33 \mathrm{~cm}$ ) was correlated with BAL. The question of whether the effect at the intermediate distance was less than at the near and far distances in Brecher et al.'s experiment is difficult to evaluate because of their qualitative treatment of their data. However, it appears that this is what in fact occurred with orthophoric subjects. Brecher et al.'s findings represent a very incomplete test. Their report lacks statistical analyses, they assessed responses at only one intermediate distance, and their experiment lacked important controls for time- and expectation-related effects on the vision variables.

On the basis of phoria measures at each of the three target distances, Brecher et al. (1955) suggested that the target distance at which no alcohol-induced heterophoria would appear would be about $56-60 \mathrm{~cm}$. Cohen and Alpern (1969) made tests of accommodative vergence at various intermediate distances and concluded that such a fixed "neutral" point could not be established. That is, the point of no phoria appeared to vary with BAL, although it was intermediate in all cases.

The goal of the present experiment was to examine systematically the relation between alcohol intoxication and fusion latency. Because of the previously described effect of alcohol in reducing the range of available vergence, it was expected that alcohol would increase fusion latency at far and near viewing distances, that the far point of fu- sion would shift toward the observer, and that the near point would shift away from the observer as intoxication level increased. If alcohol-induced diplopia resulted primarily from changes in motor fusion, with little or no impact of alcohol on sensory fusion, then fusion latency for intermediate distances would be relatively unaffected by alcohol. On the other hand, if alcohol affected sensory fusion, alcohol-induced increases in fusion latency would occur for intermediate distances as well.

\section{METHOD}

\section{Subjects}

Subjects were 8 volunteers, 21-24 years old, in good health, with no current or prior drinking problems, and no genetic or familial history of alcoholism. All were within $10 \%$ of desirable weight (Metropolitan Life Insurance Company, 1983). To avoid the complexities of sex and menstrual cycle differences in alcohol absorption and metabolism (e.g., Frezza et al., 1990; Jones \& Jones, 1976; Mishra, Sharma, Potter, \& Mezey, 1989), the sample was limited to males. All were moderate drinkers, defined by a QFA (quantityfrequency, adjusted) range of $0.25-1.5 \mathrm{oz}$ ethanol/day, using the alcohol consumption questionnaire of Armor and Polich (1982). All subjects gave informed consent to participate after the nature of the procedures had been fully explained. Each received $\$ 65$ for his participation.

The subjects were screened with a Bausch and Lomb Ortho-Rater and a Dioptron Nova diagnostic eye computer. All had fine stereopsis within normal limits (threshold stereopsis angle of $27 \mathrm{sec}$ or less), and no history of serious vision pathology. Each had uncorrected acuity of at least 0.9 , near and far, in both eyes. No subject had any measurable vertical phoria, nor lateral phoria outside the following ranges: $+2.33 \mathrm{PD}$ eso to $-1.66 \mathrm{PD}$ exo (far); 0 to $-7.5 \mathrm{PD}$ exo (near). All subjects had spherical equivalents refractive errors within the range of -1.0 to $+0.5 \mathrm{D}$, with cylinder values within -1.0 to +1.0 , for each eye.

\section{Apparatus and Environment}

Intoximeter. An Intoximeter (Mark IV, Intoximeter, Inc., St. Louis) was used for BAL estimates. This instrument uses gas chromatography to estimate BAL from deep-lung air samples.

Amblyoscope. All fusion latency measurements were made with a modified Lyle Major Amblyoscope (shown schematically in Figure 1). Each of the subjects' eyes looked into a separate viewing tube, at the end of which, $16 \mathrm{~cm}$ from the corneal apex, was a backilluminated target slide. By changing the angles of the viewing tubes, it was possible to vary the amount of vergence required for fusion of the targets. Accessory lens mounts were attached to the viewing tubes. Each target was illuminated by a 6-V Krypton bulb (Rayovac K13-2), powered by a variable power supply. Between each bulb and its slide was a Uniblitz Model 225L shutter, powered by a Uniblitz Model $300 \mathrm{C}$ control. Hunter decade interval timers and Industrial Timer Corporation stop clocks provided control over when each shutter opened and closed and a record of how long each shutter was open.

Each target slide was constructed by cutting a vertical strip, $1.5 \times 44.0 \mathrm{~mm}$, from the center of a square sheet of black paper with $82-\mathrm{mm}$ sides. The resulting opening was covered with a yellow filter. The slide was mounted on a sheet of transparent Plexiglas, $82.0 \mathrm{~mm}$ square and $3.0 \mathrm{~mm}$ thick. When the subject looked into the viewing tubes with the shutters open, each eye saw a vertical yellow line $\left(0.5^{\circ} \times 15.4^{\circ}\right.$ visual angle; approximate Munsell notation $=2.5 Y 8 / 12$ ), which, when fused, appeared as a single line in a dark field. The luminance of each line was approximately $150 \mathrm{~cd} / \mathrm{m}^{2}$. The accommodation required for optimal focus of each target (i.e., stimulus accommodation) could be controlled via the placement of trial lenses in the lens mounts. 


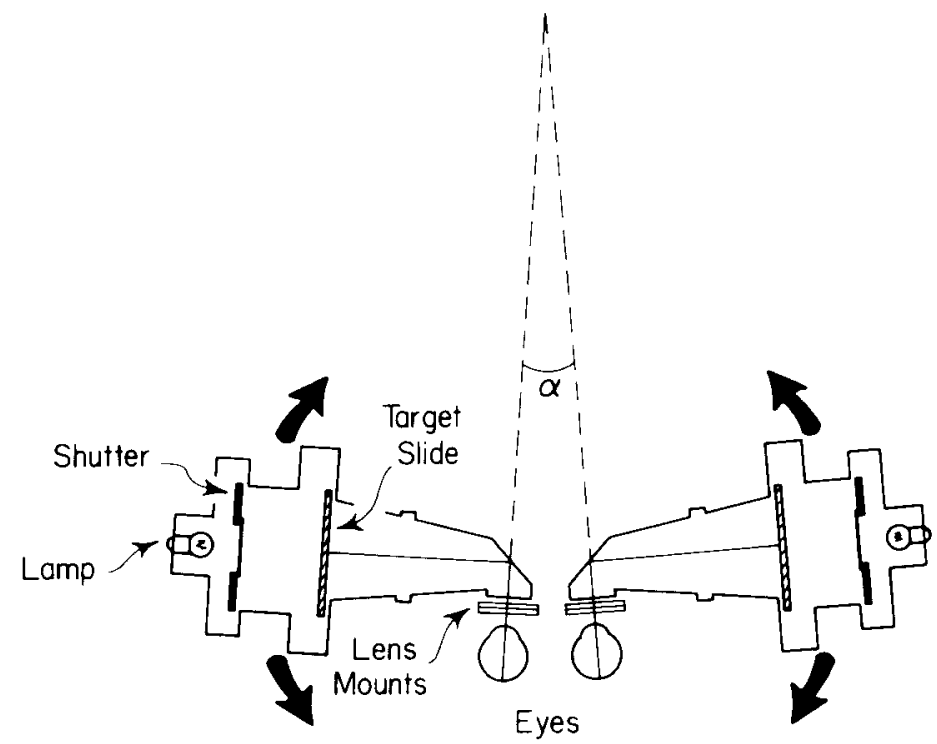

Figure 1. Schematic view of amblyoscope, as viewed from above. As indicated by the arrows, the orientation of the viewing tubes can be varied to change the vergence angle required for binocular fixation. The vergence angle is indicated as alpha.

Reaction time apparatus. Simple reaction time (RT) was assessed with an apparatus constructed of a Hunter decade interval timer, an Industrial Timer Corporation stop clock, and a Lafayette Model 1605 light box, which contained a GE F4T5 CW lamp mounted behind a translucent white Plexiglas screen. The screen was masked, so that when the lamp was illuminated a $2.6 \times 6.25 \mathrm{~cm}$ back-illuminated white rectangle appeared to the observer. The luminance of the strip, when lit, was $410 \mathrm{~cd} / \mathrm{m}^{2}$.

Testing environment. During testing, all lights were extinguished with the exception of some small lamps used by the experimenters to work by. When the subject was looking into the amblyoscope, the only light visible to him was that provided by the lines on the target slides.

\section{Procedure}

The subjects volunteered in response to a newspaper advertisement. They were mailed a description of the experiment, a consent form, a health and vision questionnaire, and the alcohol consumption questionnaire. Of those who returned the forms, several who met the health and alcohol consumption criteria participated in a screening session. Those who successfully completed the screening tasks participated in the experimental sessions.

Screening session. During the screening session, the subject's height, weight, and interpupillary distance were assessed. He was handed a tube and asked to read a Snellen chart through it; the eye that he used was recorded as his dominant eye. Then, Ortho-Rater and Dioptron Nova assessments were performed. If the subject met criteria on these measures, he practiced fusing the amblyoscope targets with a variety of vergence settings. He was instructed to avoid alcohol and other drugs on testing days, and to avoid consuming anything other than water during the $3 \mathrm{~h}$ preceding each session.

Experimental sessions. There were two experimental sessions, administered in counterbalanced order, each corresponding to an experimental condition. The procedures for each condition were identical except for the contents of the drink consumed at the beginning of the session. For the placebo condition, the drink consisted only of diluent. For the alcohol condition, the drink contained diluent plus ethanol. Each condition was run on a different day, conducted at the same time of day (the evening) to minimize diurnal effects on alcohol response.
Each experimental session began with weighing of the subject, questioning to ensure that he had consumed no alcohol, food, or medication during the proscription intervals, and an Intoximeter BAL assessment. The subject then ate a small amount of plain white bread $(0.9 \mathrm{~g} / \mathrm{kg}$ of body weight). Previous studies (e.g., Miller et al., 1985; Miller et al., 1986) have shown that this controls the occasional problem of nausea resulting from alcohol on an empty stomach without unduly suppressing BAL curves.

Next, the subject was given a drink consisting of $7.5 \mathrm{ml}$ of liquid per $\mathrm{kg}$ of body weight. It was consumed during a 20-min period, with $25 \%$ of it given every $5 \mathrm{~min}$. For the placebo (PL) condition, the drink was pure diluent. For the alcohol (AL) condition, it included $1.4 \mathrm{ml} / \mathrm{kg}$ of 190 proof (95\%) ethanol, the remainder being diluent. Each drink was served in a covered opaque cup and consumed through a straw. On the lid were two drops of ethanol and two drops of peppermint oil, making it difficult to discern the alcohol content by smell.

The diluent was made by mixing one package $(0.14 \mathrm{oz}$ or $3.97 \mathrm{~g})$ of unsweetened lemon-lime flavored Kool-Aid brand soft drink mix, 1 liter distilled water, and eight $1-\mathrm{g}(.035-\mathrm{oz})$ packets Equal brand powdered sweetener. It contained no carbonation, and little sugar or other ingredients that affect the absorption or metabolism of alcohol, and its flavor helped conceal the amount of alcohol while being acceptable to (albeit not necessarily popular with) the subjects.

At the end of the 20-min consumption period, an additional $10 \mathrm{~min}$ passed while any alcohol remaining in the subject's mouth and throat tissues was absorbed. Toward the end of this period, he rinsed his mouth thoroughly with distilled water. At the end of this period, three types of measurement were made, as described below. These measurements were repeated every $15 \mathrm{~min}$ for a total of $6 \mathrm{~h}$.

1. Blood alcohol level. The first measurement was an Intoximeter assessment of BAL.

2. Simple reaction time. The second measurement was an assessment of simple RT. The subject looked at the RT light box, positioned approximately $170 \mathrm{~cm}$ from his eyes. The experimenter initiated a 5-sec cycle of the Hunter timer. At the end of this 5-sec interval, the lamp in the light box was turned on and, concurrently, the stop clock was activated. The subject pushed a thumb-operated button switch (held in his dominant hand) as quickly as possible after the appearance of the light. The time that elapsed between 
the illumination of the lamp and the pushing of the button was recorded by the stop clock. This procedure was repeated twice, and the mean of the three values was taken as the subject's RT.

3. Fusion latency measurements. For each subject, on the basis of his interpupillary distance, it was determined which amblyoscope viewing tube angle settings (to the nearest $0.5^{\circ}$ ) would correspond to whole-number meter angle ${ }^{2}$ values of -2 through +10 . To begin, the viewing tubes were set at the vergence angle corresponding to +2 meter angles. Trial lenses of $-2 \mathrm{D}$ back vertex power were placed in the lens mounts.

The subject looked into the amblyoscope while holding a thumboperated button switch in his dominant hand. To begin the first trial, the vertical yellow bar became visible to the subject's dominant eye. At this point, there was stimulation for accommodative vergence, but not for fusional vergence. Five seconds later, the other eye's stimulus also became visible and the stop clock was activated. As soon as the subject achieved fusion, he pushed the button, which closed both shutters and blacked out the visual field. Pushing the button also stopped the stop clock, providing a reading of how much time had elapsed between the appearance of the second eye's visual stimulus and the pushing of the button. This was the measure of fusion latency. If the button was not pushed within $10 \mathrm{sec}$ of the appearance of the nondominant eye's stimulus, the shutters closed. In such cases, fusion latency was recorded as $10 \mathrm{sec}$.

For the next (second) trial, the viewing tubes were set to a new angle, corresponding to either +1 or +3 meter angles (randomly determined), appropriate trial lenses were inserted $(-1$ or $-3 \mathrm{D}$, respectively), and the procedure described above was repeated. If this second trial was for +1 meter angle, succeeding trials were for 0 , then -1 , then -2 meter angles. For each meter angle setting, trial lenses of appropriate power were inserted. At the first setting for which the subject could not achieve fusion (i.e., fusion latency $>10 \mathrm{sec}$ ), the viewing tubes were reset to the previous meter angle setting, and the viewing tube angle was decreased in $0.5^{\circ}$ steps, with a trial conducted at each step, until fusion latency was again equal to $10 \mathrm{sec}$. The vergence angle lying midway between the smallest angle for which fusion latency was less than $10 \mathrm{sec}$ and the next smaller angle on the scale defined the far point of fusion.

If, on the other hand, the second trial was for the +3 meter angle setting, subsequent trials progressed in 1-meter-angle steps toward the near point of fusion, following the same strategy as that for the far point. In reality, however, the near point almost always exceeded the 10-meter-angle limit of the apparatus.

Regardless of whether the near or the far point of fusion was approached first, once it had been determined the remaining value was then found. The subject then relaxed until the beginning of the next 15-min time period, when the BAL, RT, and fusion latency measurements described above were again made. This procedure was repeated every $15 \mathrm{~min}$ for a total of $6 \mathrm{~h}$.

In summary, there were two conditions, $\mathrm{AL}$ and $\mathrm{PL}$, each comprising 24 consecutive 15 -min time periods. For each of these time periods were obtained measures of $B A L$, simple $R T$, the near and and far points of fusion, and fusion latencies for all the meter angle settings between these two extremes.

At the end of each session, the subject's BAL was assessed periodically until it was $0.02 \%$ or below. At that point he was taken home. Each subject agreed to remain in the laboratory until his BAL had reached this criterion, and not to operate a motor vehicle or other dangerous machinery until at least $6 \mathrm{~h}$ following the session.

\section{RESULTS}

\section{Basic Blood Alcohol Level}

All BAL readings during the PL condition equaled $0 \%$, as expected. For the AL condition, the mean peak BAL for the 8 subjects was $0.105 \%(S D=0.017$, range $=$
$0.09 \%-0.14 \%$ ). The lowest BAL for the 24 time periods had a mean for the 8 subjects of $0.020 \%$ ( $S D=0.015$, range $=0 \%-0.04 \%$ ). With the time periods numbered consecutively $1-24$, the mean time period at which peak BAL was first observed was 3.63 ( $S D=2.55$, range $=$ $1-8)$. That is, peak BAL was first observed, on the average, about $40 \mathrm{~min}$ after testing began.

\section{Analyses of Near and Far Points of Fusion}

The purpose of this set of analyses was to determine whether near and far points of fusion were systematically related to intoxication level. Since both conditions required making repeated measurements for $6 \mathrm{~h}$, the first step was to determine whether there was any time-related change in near or far point in the absence of alcohol. Accordingly, near and far point values were examined across the 24 time periods for the PL condition alone. A formal analysis of near point values was unnecessary. Near point did not vary across the 24 time periods for any subject. For each time period, the mean near point was represented as $34.8^{\circ}$, defined by the 10-meter-angle limit of the apparatus.

The far point values (expressed in degrees and meter angles) for the PL condition are shown in Table 1. While the variation across time period was small in absolute terms (range $=1.38^{\circ}$ ), the analysis of variance (ANOVA) of far point expressed in degrees showed it to be statistically significant $[F(23,161)=2.55, p<.01]$. Thus, the analysis of intoxication effects on far point required a control for the possible confounding effect of time period.

To analyze the effects of intoxication on far point, each subject's far point data were divided into four blood al-

Table 1

Far Points of Fusion and Mean Fusion Latencies for the $\mathbf{2 4}$ Time Periods of the Placebo Condition

\begin{tabular}{cccc}
\hline $\begin{array}{c}\text { Time } \\
\text { Period }\end{array}$ & $\begin{array}{c}\text { Far Point } \\
\text { (Degrees) }\end{array}$ & $\begin{array}{c}\text { Far Point } \\
\text { (Meter Angles) }\end{array}$ & $\begin{array}{c}\text { Mean Fusion } \\
\text { Latency (Seconds)* }\end{array}$ \\
\hline 1 & -1.76 & -0.48 & 0.90 \\
2 & -1.50 & -0.41 & 0.77 \\
3 & -1.50 & -0.41 & 0.81 \\
4 & -1.88 & -0.51 & 0.82 \\
5 & -1.88 & -0.51 & 0.76 \\
6 & -2.12 & -0.58 & 0.74 \\
7 & -2.26 & -0.62 & 0.75 \\
8 & -2.26 & -0.61 & 0.73 \\
9 & -2.62 & -0.73 & 0.79 \\
10 & -2.62 & -0.72 & 0.73 \\
11 & -2.88 & -0.79 & 0.73 \\
12 & -2.38 & -0.65 & 0.79 \\
13 & -2.62 & -0.72 & 0.77 \\
14 & -2.38 & -0.65 & 0.74 \\
15 & -2.38 & -0.66 & 0.76 \\
16 & -2.26 & -0.62 & 0.68 \\
17 & -2.38 & -0.66 & 0.71 \\
18 & -2.76 & -0.76 & 0.73 \\
19 & -2.62 & -0.73 & 0.69 \\
20 & -2.38 & -0.66 & 0.70 \\
21 & -2.50 & -0.69 & 0.77 \\
22 & -2.76 & -0.76 & 0.72 \\
23 & -2.62 & -0.73 & 0.70 \\
24 & -2.62 & -0.72 & 0.67 \\
\hline
\end{tabular}

*Collapsed across 11 meter angle settings. 


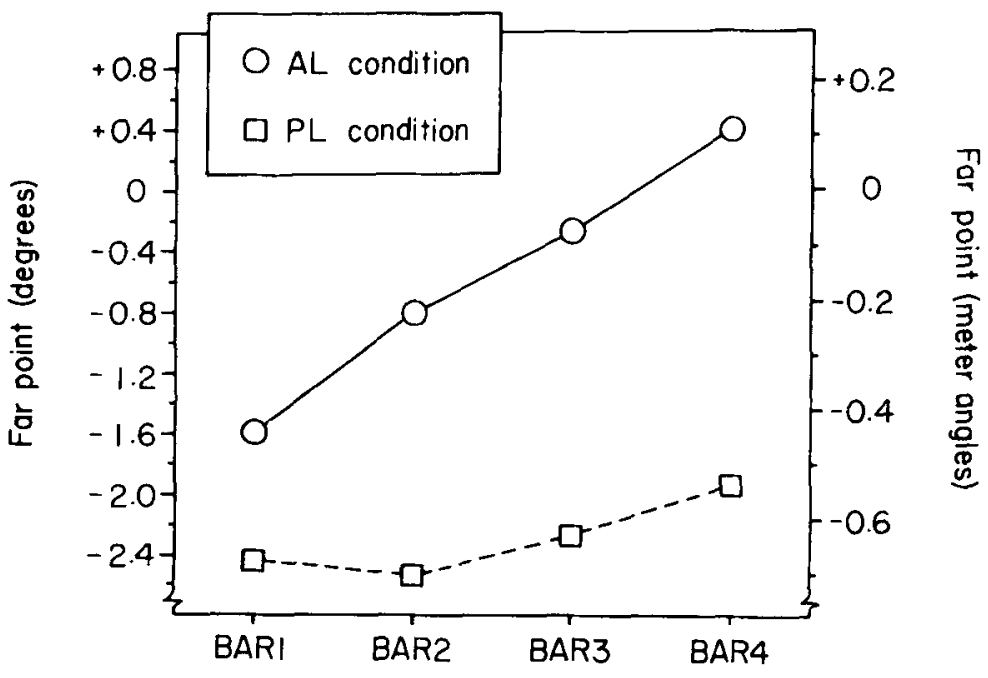

Blood alcohol range

Figure 2. Far point, expressed in degrees and meter angles, plotted as a function of blood alcohol range (BAR), for the alcohol (AL) and placebo (PL) conditions.

cohol ranges (BARs), based on BAL values observed during the $A L$ condition. For a given subject, the far point value for the first range (BAR1) consisted of the mean of all far point values obtained when his $\mathrm{BAL}=0 \%-0.04 \%$. BAR2 consisted of the mean of far point values when his BAL $=0.05 \%-0.06 \%$. BAR 3 consisted of the mean for that subject when his BAL $=0.07 \%-0.08 \%$, and BAR4 consisted of the mean when his BAL $=0.09 \%$ or greater. Far point data were expressed in the same way for the PL condition, with the four BARs defined by the same time periods as for the AL condition. Thus, for example, if Time Periods 1-3 defined BAR4 for the AL condition, the same three time periods defined BAR4 for the PL condition.

This procedure made possible a $2 \times 4$ factorial arrangement of condition (AL and PL) $\times$ blood alcohol range (BAR1, BAR2, BAR3, and BAR4), as shown in Figure 2. The ANOVA showed that the effect due to condition was significant $[F(1,7)=73.38, p<.01]$, as were the effect due to BAR $[F(3,21)=13.81, p<.01]$ and the interaction $[F(3,21)=5.39, p<.01]$. Newman-Keuls comparisons across the PL condition showed that none of the four values differed significantly. Across the AL condition, the comparison between BAR2 and BAR3 was not statistically significant. Comparisons between BAR 1 and BAR 2, and between BAR3 and BAR4, were significant $(p<.05)$. The remaining comparisons across the AL condition were significant at $p<.01$. The difference between $A L$ and PL conditions was not significant at BAR1, but was so at all other BARs $(p<.01)$.

For consistency, the analysis of the effects of BAR on near point was conducted in the same manner. The ANOVA revealed no significant effects. Indeed, only 1 subject showed a change in near point in the AL condition. It is clear that the subjects' true near points almost invariably exceeded the limits of the apparatus for both conditions.

\section{Analyses of Fusion Latency}

Since there were relatively few instances in which subjects were able to fuse the targets at meter angle settings of -1 or -2 , these settings were excluded from analysis. This left 11 fusion latency values (representing meter angle settings of 0 through +10 ) per time period. Of the resulting 4,224 fusion latency values, only $186(4.4 \%)$ exceeded $3.0 \mathrm{sec}$, and these contributed inordinately to data variability. Thus, all fusion latency values greater than $3.0 \mathrm{sec}$ were recorded as $3.0 \mathrm{sec}$ for analysis.

To determine if there was any change in fusion latency as a function of time alone, an $11 \times 24$ (meter angle setting $\times$ time period) ANOVA was performed for the fusion latency data from the PL condition. The effect of meter angle setting was significant $[F(10,70)=2.86$, $p<.01]$, as was that of time period $[F(23,161)=1.61$, $p=.047]$. The interaction was not significant. Mean fusion latency values for the 11 meter angle settings (collapsed across time period) are shown in Table 2. It is not

Table 2

\section{Mean Fusion Latencies for the 11 Meter Angle Settings} of the Placebo Condition

\begin{tabular}{cc}
$\begin{array}{c}\text { Meter Angle } \\
\text { Setting }\end{array}$ & $\begin{array}{c}\text { Mean Fusion } \\
\text { Latency (Seconds)* }\end{array}$ \\
\hline 10 & 0.75 \\
9 & 0.71 \\
8 & 0.68 \\
7 & 0.69 \\
6 & 0.67 \\
5 & 0.71 \\
4 & 0.77 \\
3 & 0.70 \\
2 & 0.60 \\
1 & 0.78 \\
0 & 1.18 \\
\hline
\end{tabular}

*Collapsed across 24 time periods. 
surprising that fusion latency was somewhat greater at the 0 -meter-angle setting than at the other settings, since this setting was close to the far point and was thus pushing divergence to its limit.

The principal reason for this analysis was to determine whether time might be confounded with intoxication level in the AL condition. Although there was a significant effect due to time period in the PL condition, the effect was just barely significant, and it rested almost entirely on the fact that fusion latency values were somewhat higher for the first time period than for the other time periods (see Table 1). When the analysis was repeated with the first time period removed, there was no significant effect due to time period. Furthermore, the first time period was not confounded with intoxication level; each of the BARs observed in the AL condition appeared with about equal frequency in the first time period. Given these observations, it was concluded that there was no danger of confounding time with intoxication level, and subsequent analyses were based on fusion latency data from the AL condition alone.

To analyze the effects of intoxication on fusion latency, the fusion latency values obtained in the $\mathrm{AL}$ condition were divided into four BARs, as described previously. This procedure made possible a $4 \times 11$ factorial arrangement of BAR (BAR1, BAR2, BAR3, and BAR4) $\times$ meter angle setting (meter angles 0-10), as shown in Figure 3.

The effect of meter angle setting was significant $[F(10,70)$ $=7.14, p<.01]$, as was that of $\operatorname{BAR}[F(3,21)=28.39$, $p<.01]$. The interaction was also significant $[F(30,210)$ $=3.43, p<.01]$. Newman-Keuls analyses were performed at each meter angle setting. At 10 meter angles, BAR1 differed from BAR3 and BAR4 $(p<.05)$. At 9 meter angles, BAR1 differed from $\operatorname{BAR} 3(p<.05)$, and from BAR4 $(p<.01)$; and BAR2 differed from BAR4 $(p<.05)$. At 1 meter angle, BAR1 differed from BAR4 $(p<.05)$. At the 0 -meter-angle setting, all BAR values differed from each other $(p<.01)$. None of the remaining comparisons was significant.

\section{Analyses of Simple Reaction Time}

Simple RT was included in the present experiment as a matter of control. If fusion latency changed as a function of intoxication, the change might not be specific to fusion. That is, it could be that alcohol produced an overall slowing of RT, and this would be reflected on any task involving an RT component. Some investigators have suggested that alcohol produces a slowing of simple RT, but others have found no effect (e.g., Carpenter, 1962; Moskowitz, 1973; Moskowitz et al., 1972).

As with previous analyses, the first step was to determine whether there were significant effects of time period alone on RT. A one-way ANOVA of RT values across the 24 time periods of the PL condition showed a small but significant effect $[F(23,161)=1.71, p=.029]$. RT values observed during the first 6-7 time periods were somewhat larger than those during later time periods. This effect seemed large enough statistically to require a control for time effects when the effects of intoxication on

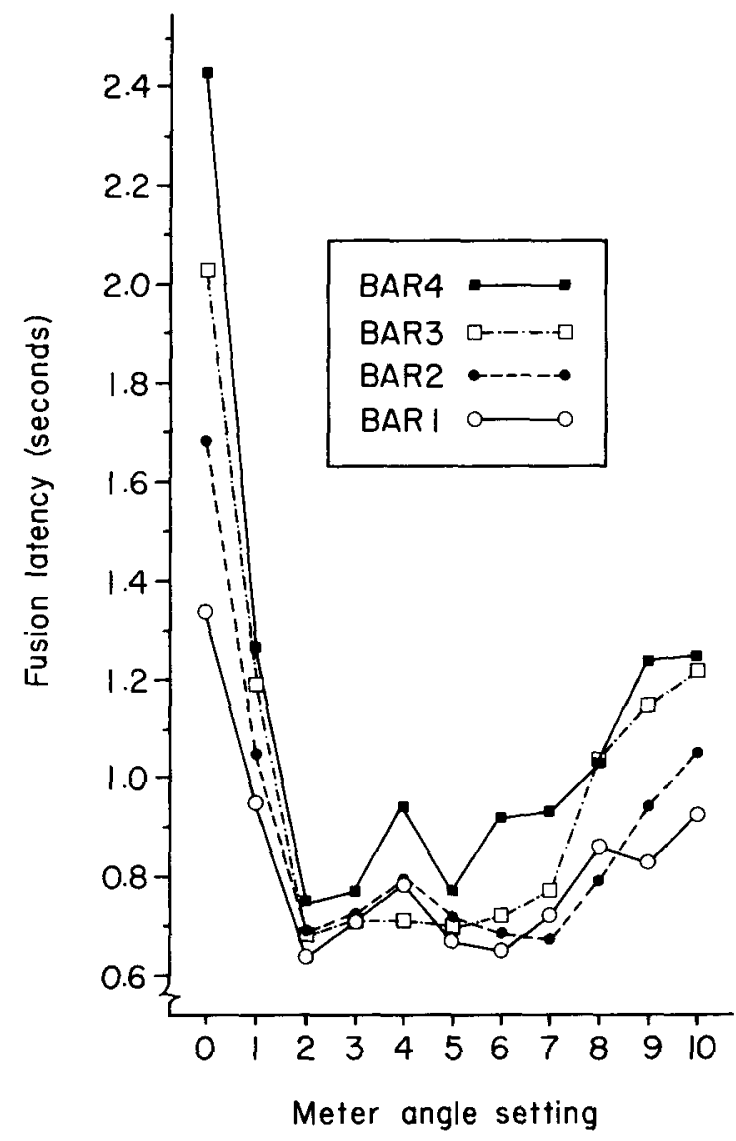

Figure 3. Fusion latency, expressed in seconds, plotted as a function of the 11 meter-angle settings, for the four blood alcohol ranges (BAR).

RT were analyzed. However, its absolute magnitude was small (the largest difference in RT between time periods was $0.06 \mathrm{sec}$ ) relative to the effects of intoxication on fusion latency (see Figure 3). Thus it appeared unnecessary to consider time-related changes in RT when the effects of intoxication on fusion latency were analyzed.

A $2 \times 4$ (condition $\times$ BAR) ANOVA was performed on the RT data, with BAR defined as in previous analyses (see Figure 4). The effect of BAR was significant $[F(3,21)=8.23, p<.01]$, as was the interaction $[F(3,21)$ $=4.71, p=.012]$. The effect of condition was not significant. Newman-Keuls analysis showed that for the $\mathrm{AL}$ condition, BAR1 differed significantly from BAR3 and BAR4 $(p<.01)$, and BAR2 differed from BAR3 $(p<.05)$ and from BAR4 $(p<.01)$. The remaining comparisons for the $\mathrm{AL}$ condition, all comparisons for the PL condition, and all comparisons of $\mathrm{AL}$ with $\mathrm{PL}$ at each of the four levels of BAR were nonsignificant. Thus, RT tended to increase with intoxication level. However, the difference in RT between BAR1 and BAR4 for the AL condition was only $0.047 \mathrm{sec}$. Thus, while RT was technically confounded on fusion latency in comparisons across BAR, its effect was so small in absolute terms that it was deemed unimportant. 


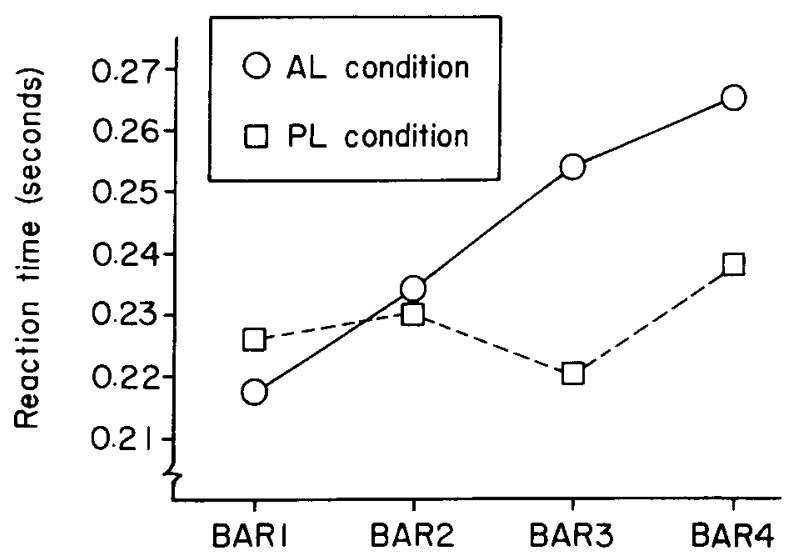

Blood alcohol range

Figure 4. Simple reaction time, expressed in seconds, plotted as a function of blood alcohol range (BAR), for the alcohol (AL) and placebo (PL) conditions.

\section{Correlational Analyses}

As an additional means of examining the relations between intoxication and the variables of interest in this study, the Pearson correlation coefficients between BAL and all of these other variables were calculated, for each subject individually, across the 24 time periods of the AL condition. The mean correlation coefficients for the 8 subjects are shown in Table 3. These correlational findings are consistent with relations shown in earlier analyses.

\section{DISCUSSION}

As expected, alcohol caused the far point to approach the observer, and the magnitude of this approach increased with intoxication. People who drink sufficient alcohol to raise their BAL above the legal limit for motor vehicle operation can expect to experience diplopia for far targets and can probably expect further that the distance at which they can see without diplopia will continue to decrease as they become more intoxicated. This has clear

Table 3

Mean Pearson Correlation Coefficients $(r)$ Derived from Comparisons of Blood Alcohol Level with Selected Other Variables Across 24 Time Periods of the Alcohol Condition

\begin{tabular}{ll}
\hline \multicolumn{1}{c}{ Variable } & Mean $r$ \\
\hline Fusion latency at 10 meter angles & $.37^{*}$ \\
Fusion latency at 9 meter angles & $.42^{*}$ \\
Fusion latency at 8 meter angles & $.46^{*}$ \\
Fusion latency at 7 meter angles & .24 \\
Fusion latency at 6 meter angles & .25 \\
Fusion latency at 5 meter angles & .26 \\
Fusion latency at 4 meter angles & .14 \\
Fusion latency at 3 meter angles & .10 \\
Fusion latency at 2 meter angles & .14 \\
Fusion latency at 1 meter angle & $.38^{*}$ \\
Fusion latency at 0 meter angles & $.55 \dagger$ \\
Simple reaction time & $.36^{*}$ \\
Far point of fusion & $.61 \dagger$ \\
\hline
\end{tabular}

${ }^{*} p<.05 . \quad+p<.01$. implications for those who drink and drive, particularly with regard to their ability to see road signs, pedestrians, other vehicles, and so forth, at a distance.

It seems clear that the near point was not tested in this experiment. A calculated risk was taken, because the amblyoscope was not readily adaptable to induce vergence closer than 10 meter angles. The findings of Brecher et al. (1955), Hogan and Linfield (1983), and Powell (1938) suggested that near points at the BALs used in this experiment would be in the general vicinity of 10 meter angles, and it was hoped that the present apparatus would be sufficient to detect alcohol-induced change. Evidently it was not, although 1 subject did show a measurable alcohol-induced outward shift in near point.

The principal analyses for this experiment were those of fusion latency. Alcohol induced an increase in fusion latency for near and far targets, with no effect at intermediate distances. Furthermore, as intoxication increased, so did the effect, with fusion latency growing progressively longer for near and far viewing. It could be expected that if intoxication continued to increase, the curves in Figure 3 would become progressively more $\mathrm{V}$ shaped, with the point of the V located at 2-3 meter angles. Such an expectation remains to be tested, of course, but it is consistent with the conception of an intermediate "neutral" point of vergence, which is perhaps synonymous with the intermediate tonic position described by Owens and Leibowitz (1983).

It should be remembered that the fusion latency changes shown in Figure 3 were mostly at distances at which fusion was still attainable. People who drink alcohol can expect that their fusional ability will be affected even when diplopia is not evident. Tasks requiring rapid target detection and recognition (e.g., responding to unexpected objects on the highway) could be affected, because even though such targets could be fused, doing so could require more time if the viewer was intoxicated rather than sober. It is clear that targets do not have to be very far away to produce such an effect-indeed, significant effects were observed as close as 1 meter angle.

It should also be noted that this experiment was a very conservative test. The levels of intoxication were relatively low, rarely exceeding most states' legal limits for the operation of motor vehicles. The fusion task was designed to make fusion as easy as possible to attain. That is, each target provided a stimulus for accommodation that was consistent with its vergence demand, and the subject had sufficient time for accommodative vergence to occur before fusional vergence was required. Furthermore, each target required only a small vergence increment or decrement relative to the target that preceded it. This is quite unlike situations in the real world, in which attention often shifts quickly from near to far targets or vice versa, requiring large shifts in accommodative and fusional vergence. Nevertheless, significant effects of alcohol on fusional ability were observed, even (at 0 meter angles) for BAL values as low as $0.05 \%-0.06 \%$. Such findings argue that the usual legal limit of $0.10 \% \mathrm{BAL}$ for motor vehicle operation is a liberal one. 
The use of a placebo condition yielded confidence that the effects were due to alcohol, rather than to time-related changes resulting from fatigue, boredom, practice, and so forth, or to subjects' expectations regarding the effects of alcohol. The subjects were led to believe that the placebo drink contained alcohol, and self-ratings confirmed that they believed this. Such controls are rarely used in studies of alcohol and vision, and they add considerably to the difficulty and expense of such research. The requirements of good experimental design demanded that they be incorporated, however.

On a more theoretical level, the following account is tentatively offered. Alcohol induces a general inhibition of both convergence and divergence, perhaps via depression of vergence mechanisms in the brainstem reticular formation. This results in a functional narrowing of the range of available vergence, such that near convergence and far divergence become more difficult. As a result, motor fusion at extreme near and far distances becomes more difficult as intoxication level increases, resulting in an increase in fusion latency and eventually to loss of fusion.

Such an account is, of course, extremely simplistic, and it requires substantiation on many levels. It requires, for example, the presence of convergence and divergence neurons. This is a point of considerable debate, but there is evidence favoring the existence of such neurons in the mesencephalic reticular formation (see, e.g., Mays, 1984). Also required is evidence that alcohol affects such neurons; it is widely believed that alcohol not only affects the reticular activating system, but that this is one of the first brain systems affected by even small amounts of alcohol (see, e.g., Blum, 1982; Himwich \& Callison, 1972; Ritchie, 1985), a fact consistent with the observation in the present experiment that even relatively low levels of intoxication show measurable effects on fusion latency. If there is such an effect of alcohol on the vergence system, it would lead to the expectation of a generalized effect on vergence, not specific to fusion. Various studies have shown that the range of both accommodative and fusional vergence is reduced by alcohol (e.g., Miller et al., 1986). Finally, if the effect of alcohol on diplopia is the result of changes in motor fusion, and not sensory fusion, one would expect that fusion would not be affected for intermediate-distance targets. The results of the present experiment are consistent with such an expectation.

The results of the present experiment can only be treated as consistent with the above account. They certainly do not substantiate it, and alternative explanations are possible. One explanation might be that alcohol reduces the relative effectiveness of the stimulus to fusion, thereby biasing subjects' vergence toward their phoria position. That is, alcohol-induced changes might be due to a reduction of the efficacy of the sensory fusion system, such that vergence tends toward the phoria position. Although alcohol-induced changes in phoria were not assessed in the present experiment, available evidence shows that there is an alcohol-induced shift in phoria toward an in- termediate (possibly drifting) neutral value that would be consistent with such an account (Brecher et al., 1955; Cohen \& Alpern, 1969). Such an explanation cannot be dismissed on the basis of the present findings, although it seems likely that if alcohol produced some generalized reduction in the effectiveness of the stimulus to fusion, changes in fusion latency also would have been detected at intermediate distances.

Fusion latency was measured subjectively in the present experiment, and there were no measures of alcoholinduced changes in vergence or fixation disparity. Thus, the conclusion that the change in fusion latency can be accounted for in terms of a restricted vergence range is an inference based on previous studies. However, such studies have shown that alcohol affects these phenomena in a manner consistent with this inference. That is, as mentioned previously, as alcohol is ingested, vergence range is reduced and fixation disparity is at its maximum for near and far targets.

\section{REFERENCES}

Adams, A. J. (1978). Acute effects of alcohol and marijuana on vision. In S. J. Cool \& E. L. Smith (Eds.), Frontiers in visual science (pp. 93105). New York: Springer-Verlag.

Armor, D. J., Polich, J. M. (1982). Measurement of alcohol consumption. In E. M. Pattison \& E. Kaufman (Eds.), Encyclopedic handbook of alcoholism (pp. 72-80). New York: Gardner.

BLUM, K. (1982). Neurophysiological effects of alcohol. In E. M. Pattison \& E. Kaufman (Eds.), Encyclopedic handbook of alcoholism (pp. 105-134). New York: Gardner.

Brecher, G. A., Hartman, A. P., \& Leonard, D. D. (1955). Effect of alcohol on binocular vision. American Journal of Ophthalmology, 39 (No. 2, Pt. II), 44-52.

Carpenter, J. A. (1962). Effects of alcohol on some psychological processes: A critical review with special reference to automobile driving skill. Quarterly Journal of Studies on Alcohol, 23, 274-314.

Cline, D., Hofstetter, H. W., \& Griffin, J. R. (1989). Dictionary of visual science (4th ed.). Radnor, PA: Chilton.

CoHen, M. M., ALPERN, M. (1969). Vergence and accommodation: VI. The influence of ethanol on the AC/A ratio. Archives of Ophthalmology, 81, 518-525.

Colson, Z. W. (1940). The effect of alcohol on vision: An experimental investigation. Journal of the American Medical Association, 115, 1525-1527.

DUKE-ElDeR, S., \& WyBAR, K. (1973). System of ophthalmology: Vol. 6. Ocular motility and strabismus. St. Louis: Mosby.

Frezza, M., DiPadova, C., Pozzato, G., Terpin, M., Baraona, E., \& Lieber, C. S. (1990). High blood alcohol levels in women: The role of decreased gastric alcohol dehydrogenase activity and first-pass metabolism. New England Journal of Medicine, 322, 95-99.

Himwich, H. E., Callison, D. A. (1972). The effects of alcohol on evoked potentials of various parts of the CNS of the cat. In B. Kissin \& H. Beglieter (Eds.), The biology of alcoholism: Vol. 2. Physiology and behavior (pp. 67-84). New York: Plenum.

Hogan, R. E., \& Linfield, P. B. (1983). The effects of moderate doses of ethanol on heterophoria and other aspects of binocular vision. Ophthalmic \& Physiological Optics, 3, 21-31.

JoNES, B. M. , JoNES, M. K. (1976). Women and alcohol: Intoxication, metabolism, and the menstrual cycle. In M. Greenblatt \& M. A. Schuckit (Eds.), Alcoholism problems in women and children (pp. 103136). New York: Grune \& Stratton.

MAYS, L. E. (1984). Neural control of vergence eye movements: Convergence and divergence neurons in midbrain. Journal of Neurophysiology, 51, 1091-1108.

McNamee, J. E., Piggins, D., \& Tong, J. (1981). Confirmation of 
the influence of alcohol on heterophoria using a vision screener. American Journal of Optometry \& Physiological Optics, 58, 761-765

Metropolitan Life. Insurance Company (1983). 1983 Metropolitan height and weight tables. Statistical Bulletin, 64, 2-9.

Miller, R. J., Pigion, R. G., Martin, K. D. (1985). The effects of ingested alcohol on accommodation. Perception \& Psychophysics, $37,407-414$

Miller, R. J., Pigion, R. G., Takahama, M. (1986). The effects of ingested alcohol on accommodative, fusional, and dark vergence. Perception \& Psychophysics, 39, 25-31.

Mishra, L., Sharma, S., Potter, J. J., \& Mezey, E. (1989). More rapid elimination of alcohol in women as compared to male siblings. Alcoholism: Clinical \& Experimental Research, 13, 752-754.

Moskowitz, H. (1973). Laboratory studies of the effects of alcohol on some variables related to driving. Journal of Safety Research, 5 , 185-199.

Moskowitz, H., Sharma, S., \& Shapero, M. (1972). A comparison of the effects of marijuana and alcohol on visual functions. In M. F. Lewis (Ed.), Current research in marijuana (pp. 129-150). New York: Academic Press.

OWEns, D. A., \& Leibowitz, H. W. (1983). Perceptual and motor consequences of tonic vergence. In C. M. Schor \& K. J. Ciuffreda (Eds.), Vergence eye movements: Basic and clinical aspects (pp. 25-74). Boston: Butterworths.

PoWELl, W. H., JR. (1938). Ocular manifestations of alcohol and a consideration of individual variations in seven cases studied. Jourmal of Aviation Medicine, 9, 97-103

Ritchie, J. M. (1985). The aliphatic alcohols. In A. G. Gilman, L. S. Goodman, T. W. Rall, \& F. Murad (Eds.), Goodman and Gillman's The pharmacological basis of therapeutics (7th ed., pp. 372-386). New York: Macmillan.
SEEDORFF, H. H. (1956). Effect of alcohol on the motor fusion reserves and stereopsis as well as on the tendency to nystagmus. Acta Ophthalmologica, 34, 273-280.

WILson, G., MrTChell, R. (1983). The effect of alcohol on the visual and ocular motor systems. Australian Joumal of Ophthalmology, 11. 315-319.

ZUSCHLAG, H. G. (1965). Alkoholbedingte Störungen von Sehfunktionen. Klinische Monatsblaner fur Augenheillkunde, 147, 549-600.

\section{NOTES}

1. Blood alcohol level (BAL) is a standard indication of the amount of alcohol in the blood. A frequent alternative term is blood alcohol concentration, or BAC. The most common expression of BAL is in gram percent $(\mathrm{g} \%)$, which refers to grams of alcohol per $100 \mathrm{ml}$ of blood plasma, converted to a percentage. Thus, a subject whose blood contains $0.1 \mathrm{~g}$ alcohol per $100 \mathrm{ml}$ blood would have a BAL of $0.10 \%$, the value that defines the legal limit in most states for operating a motor vehicle.

2. One meter angle is the amount of vergence required when the eyes are binocularly fixating a point on the median line at a distance of $1 \mathrm{~m}$. The vergence angle that this represents varies, of course, with interpupillary distance. The distance of the point to which the observer is verged (in meters) is thus equal to the reciprocal of meter angles, just as with diopters of accommodation. For further details, see Duke-Elder and Wybar (1973) or Cline, Hofstetter, and Griffin (1989).

(Manuscript received December 7, 1990; revision accepted for publication July 25,1991 .) 